\title{
Frequency Locking in Spatially Extended Systems
}

\author{
Hwa-Kyun Park* \\ Department of Physics,Korea Advanced Institute of Science and Technology,Taejon 305-701,Korea
}

(25 April 2000)

\begin{abstract}
A variant of the complex Ginzburg-Landau equation is used to investigate the frequency locking phenomena in spatially extended systems. With appropriate parameter values, a variety of frequency-locked patterns including flats, $\pi$ fronts, labyrinths and $2 \pi / 3$ fronts emerge. We show that in spatially extended systems, frequency locking can be enhanced or suppressed by diffusive coupling. Novel patterns such as chaotically bursting domains and target patterns are also observed during the transition to locking.
\end{abstract}

PACS numbers: 47.54.+r, 82.40.Ck, 82.20.Mj

Typeset using REVTEX

*e-mail: childend@front.kaist.ac.kr

TEL:+82-42-869-2563ＦA: +82-42-869-2510 
Frequency locking of nonlinear oscillators is a well known phenomenon, and has been studied extensively [1.2]; however, frequency locking in spatially extended systems is not well understood. Recently, frequency locking has been observed experimentally in the light sensitive Belousov-Zhabotinsky reaction [3:4]. The system is oscillatory, and shows spiral patterns without external forcing. When periodically illuminated with light pulses, however, it exhibits frequency-locked patterns such as flats, $\pi$ fronts, labyrinths and $2 \pi / 3$ fronts, depending on the forcing frequency. It was also reported that these resonances have the structure similar to Arnol'd tongues [5].

For the theoretical investigation of frequency locking in spatially extended systems, we consider the forced complex Ginzburg-Landau equation [6 [13

$$
\partial_{t} \psi=(1-i \nu) \psi+(-1+i b)|\psi|^{2} \psi+(1+i d) \nabla^{2} \psi+c \bar{\psi}^{n-1},
$$

where $\psi(\vec{r}, t)$ is a complex order parameter, and $\nu, b, d, c$ are real.

A theoretical study on the Eq. (1) showed that resonant forcing can exhibit rich patterns as varying the external frequency [6]. The same equation has been studied for parametric resonance cases in the system such as Faraday waves [7,8] and vertically oscillated granular layers [9]. However these systems are turbulent [6] or stationary [0] 9] without forcing. For the oscillatory cases, the studies have been focused on the front dynamics in 2:1 [10] and $2 n: 1$ [11 [13] resonance cases. More works are required to understand the frequency locking phenomena in spatially extended system.

In this letter, we analyze the Arnol'd tongue structures of spatiotemporal patterns for 1:1,2:1 and 3:1 resonance cases. Especially, we describe how diffusive couplings affect the frequency locking.

Firstly, we will explain the model and parameter values. Eq. (1) with $c=0, \nu=0$ is a well-known complex Ginzburg-Landau equation, which describes oscillatory media and shows stably rotating spiral patterns when $1-b d>0$ [14 [16]:

$$
\partial_{t} \psi=\psi+(1+i d) \nabla^{2} \psi+(-1+i b)|\psi|^{2} \psi
$$


In this case, the model is considered as the amplitude equation of chemical oscillations $h_{0}=\psi e^{i w t}+$ c.c., where $w$ is the frequency of a spiral rotation in real experiments.

Introducing a local amplitude $A(\vec{r}, t)$ and a local phase $\phi(\vec{r}, t)$ by $\psi(\vec{r}, t)=$ $A(\vec{r}, t) \exp (i \phi(\vec{r}, t))$, Eq. (2) reduces to the following phase equation [14, 15]:

$$
\partial_{t} \phi=b+(1-b d) \nabla^{2} \phi-(b+d)(\nabla \phi)^{2} .
$$

It has a oscillatory solution $\phi=\left(b-(b+d) k^{2}\right) t+\vec{k} \cdot \vec{r}+\phi_{0}$. Diffusion coefficient $1-b d$ must be positive for stable phase dynamics. Therefore, two constraints on parameter values, $1-b d>0$ and $b+d<0$, are required from the experimental facts that without forcing there are stably rotating spiral patterns and its frequency is higher than that of a homogeneous system [3]. Specifically, we choose $b=-0.5, d=-1.4$.

Now, consider when the spirals are forced with external frequency $w_{e}=n(w+\nu)$, where $n$ is an irreducible integer fraction and $\nu$ is a small deviation from a rational multiple of the natural frequency $w$. In this case, $\psi$ is taken as the complex amplitude of chemical oscillation $h=\psi e^{i(w+\nu) t}+$ c.c., so that stationary $\psi$ may indicate the entrainment of system to the external frequency $w_{e}$. Correspondence between $h$ and $h_{0}=\psi e^{i w t}+$ c.c. when the forcing is absent gives the term $-i \nu \psi$ in Eq. (四). Considering the invariance of the system under a translation $t \rightarrow t+\frac{2 \pi}{w_{e}}$ yields the resonant forcing term $c \bar{\psi}^{n-1}$ in Eq. (1)

Next, we will obtain the Arnol'd tongue structure of Eq. (1) and study the characteristic of resonant patterns. Eq. (11) contains two control parameters $\nu$ (measuring frequency mismatch) and $c$ (forcing amplitude). In this parameter space, we seek the stationary solutions of Eq. (1), which means frequency locking. Without diffusion the linear stability analysis of the stationary state yields the Arnol'd tongue of a single oscillator (given as solid lines in Fig.(1).

There are $n$ equivalent stable stationary solutions for $n: 1$ resonances. These solutions have the same amplitudes, but are phase-shifted with respect to each other. In spatially extended systems, different oscillators may lock to $n$ different states to make $2 \pi / n$ front solutions. 
To obtain the resonance regions of spatiotemporal patterns, we integrate Eq. (1) numerically. System size is 100 by 100 with periodic boundary conditions. The initial condition is a set of Gaussian distributed random numbers of mean zero, and variance 0.25. The numerical results are summarized in Fig.1; the typical patterns are presented in Fig.2.

When the frequency locking does not occur, basic patterns are spirals. At 1:1 resonance, resonant patterns are homogeneous flats (Fig.2(a)). At 2:1 resonance, there are two resonant patterns: $\pi$ fronts (Fig.2(b)) and labyrinthine patterns (Fig.2(d)). Fig.11(b) shows that labyrinthine patterns arise at higher frequencies than $\pi$ fronts. Under some conditions $\pi$ fronts move until only one domain remains. These front dynamics and the nonequilibrium Ising-Bloch transition(NIB) have been studied by previous researchers [5, 10,17]. At 3:1 resonance, we can see the $2 \pi / 3$ fronts as in Fig.2(c). But the time series at one typical point show that these fronts are in fact not stationary, but move slowly(Fig.3(a)). The periodic nature of time series indicates the motion of the fronts. The flat region of time series corresponds to the time interval during which each domain passes. Sometimes moving fronts merge and eventually collapse into flat patterns. Ref . [10] shows that chiral interfaces move in nonequilibrium systems. In this respect, observed $2 \pi / 3$ front motion can be explained from the nonzero chirality of the interfaces (Fig. 3(b)).

Next, we discuss how diffusion affects frequency locking and Arnol'd tongue structures (See Fig.11). The regions indicated by 1F, 2F, 3F and LABY, which corresponds to flat, $\pi$ fronts, $2 \pi / 3$ fronts and labyrinths, respectively, are frequency locked. The Arnol'd tongue structure in Eq. (11) almost coincides with that of a single oscillator, but some deviations are found. For example, the tip of Arnol'd tongue is smoothed so that locking begins at a nonzero forcing amplitude. It is rather surprising that frequency-locked labyrinths arise at parameter values at which a single oscillator without coupling would not be locked. It means that diffusion induces frequency locking. One can also see that at lower $\nu$, the locking is somewhat suppressed. (Note that the regions indicated by BD are not frequency-locked.)

To understand the enhancement and suppression of locking, we consider the phase dynamics of Eq. (1). Substituting $\psi(\vec{r}, t)=A(\vec{r}, t) \exp (i \phi(\vec{r}, t))$ into Eq. (1) and assuming 
that the amplitude is slaved to the phase, we get the following phase equation for $n=2$ :

$$
\partial_{t} \phi=(b-\nu)+(1-b d) \nabla^{2} \phi-(b+d)(\nabla \phi)^{2}+c \sqrt{1+b^{2}} \cos (2 \phi+\delta),
$$

where $\tan (\delta)=1 / b$.

Eq. (四) has an oscillatory solution $\phi=\left(b-\nu-(b+d) k^{2}\right) t+\vec{k} \cdot \vec{r}+\phi_{0}$ for $c=0$. The stationary solution for nonzero c means the entrainment of the system to the forcing frequency: frequency locking. There are two effects on the frequency of system: forcing and nonlinear coupling [19]. Forcing, represented by $c \sqrt{1+b^{2}} \cos (2 \phi+\delta)$, compensates the term $(b-\nu)$ and entrains the system. Thus, without the nonlinear coupling $-(b+d)(\nabla \phi)^{2}$, a frequency-locked stationary solution occurs for $c>|b-\nu| / \sqrt{1+b^{2}}$. In other words, forcing increases the frequency for $b-\nu<0$ and decreases the frequency for $b-\nu>0$. However, nonlinear coupling always increases the frequency of the system by $-(b+d) k^{2}[20]$. Hence, for high $\nu$, forcing and nonlinear coupling effects are cooperating and frequency locking is enhanced. But for low $\nu$, two effects are competing and locking is suppressed.

These effects also explain the following transition routes to locking as increasing the external forcing amplitude c. (See Fig.1)

low $\nu:$ spiral $\rightarrow$ chaotically bursting domain $\rightarrow$ frequency-locked front

high $\nu:$ spiral $\rightarrow$ labyrinth $(n=2)$, target $(n=3) \rightarrow$ frequency-locked front

At low $\nu$, the turbulent states, characterized by chaotic nucleation and annihilation of domains, arise between spirals and locked states(Fig.2(f)). As explained above, the diffusion effect against locking increases with $(\nabla \phi)^{2}$ at low $\nu$. Near the defect core, $(\nabla \phi)^{2}$ is large and frequency locking is suppressed. However, far from the defect core, $(\nabla \phi)^{2}$ is small and domains are frequency-locked. As the defects and the domains move, the dynamics become quite complex. The chaotic time series of $\operatorname{Re}(\psi)$ at one typical point, shown in Fig.t(a), consists of flat parts and irregular oscillating parts. Time evolution of $(\nabla \phi)^{2}$ at the same point is also given in Fig. (4) (b). One can observe that irregular oscillating parts correspond to large $(\nabla \phi)^{2}$. Further increasing the external forcing, the domain pervades the entire region and frequency-locked patterns form. 
At high $\nu$, increasing the amplitude $c$ gives labyrinths or targets. The labyrinths have a finite $(\nabla \phi)^{2}$, which helps the frequency locking now. So, locked labyrinths can arise outside the Arnol'd tongue of a single oscillator. But the targets at 3:1 resonance are not frequency locked. Although it might be due to the chirality, the origin of unlocked target patterns is not clear at this stage and should be studied further.

In summary, we examined frequency-locking phenomena in spatially extended systems using a complex Ginzburg-Landau equation with a resonant term. A resonant tongue structure was constructed and studied as a function of $\nu$ (measuring frequency mismatch) and $c$ (amplitude of forcing). Analysis with the phase equation showed that frequency locking is enhanced by diffusion at higher forcing frequencies, but suppressed at lower forcing frequencies. This explains the locus of locked labyrinths and unlocked chaotically bursting domains in parameter space with respect to Arnol'd tongues.

The results are in agreement with recent experimental reports [3 5]. For example, labyrinths formed at higher frequencies than $\pi$ fronts; $2 \pi / 3$ fronts at $3: 1$ resonance move due to the chirality. In addition, novel patterns such as chaotically bursting domains and target patterns were also observed during the transition to locking. It would be interesting to know whether these are confirmed experimentally as well.

The author wishes to thank H.-T.Moon, S.-O.Jeong and T.-W.Ko for many stimulating discussions. This work was supported in part by the project HPC-COSE from the Ministry of Science and Technology and in part by BK21 program of the Ministry of Education in Korea. 


\section{REFERENCES}

[1] E. Ott, Chaos in Dynamical Systems (Cambridge,1993)

[2] P. Manneville, Dissipative Structures and Weak Turbulence (Academic,1990)

[3] V. Petrov, Q. Ouyang, and H. L. Swinney, Nature. 388, 655 (1997).

[4] A. L. Lin et al., in Pattern formation in Continuous and coupled systems edited by M.Golubitsky, Dan Luss, and S.H.Strogatz.(Springer-Verlag,1999)

[5] A. L. Lin, M. Bertram, K.Martinez and H. L. Swinney, Phys. Rev. Lett. 84, 4240 (2000).

[6] P. Coullet and K. Emilson, Physica (Amsterdam) 61D, 119 (1992).

[7] W. Zhang and J. Vinals, Phys. Rev. Lett. 74, 690 (1995).

[8] P. Coullet, T. Fishchi, and G. Sonnio Phys. Rev. E 49, 2087 (1994).

[9] L. Tsimring and I. Aranson, Phys. Rev. Lett. 79, 213 (1997).

[10] P. Coullet and J. Lega, Phys. Rev. Lett 65, 1352 (1990).

[11] C. Elphick and A. Hagberg, Phys. Rev. Lett. 80, 5007 (1998).

[12] C. Elphick and A. Hagberg, Phys. Rev. E. 59, 5285 (1999).

[13] A. L. Lin, A.Ardelea, M.Bertram, H.L.Swinney and E.Meron, Phys. Rev. E. 62, 3790 (2000)

[14] Y. Kuramoto, Chemical Oscillations, Waves and Turbulence (Springer-Verlag,1984)

[15] A.S.Mikhailov, Foundations of Synergetics I (Springer-Verlag 1990)

[16] H. Chaté and P. Manneville, Physica (Amsterdam) 224A, 348 (1996)

[17] V. Petrov, M. Gustafsson, and H. L. Swinney in Proceedings of the 4th Experimental Chaos Conference edited by M.Ding et al.

[18] K. Kaneko, Physica (Amsterdam) 54D, 5 (1991). 
[19] Both the linear and nonlinear coupling term in Eq. (4) come from the diffusion term in Eq. (1). Note that the linear term in Eq. (四) does not contribute to the frequency significantly since the phase variable $\phi$ takes a form like $\phi \sim \vec{k} \cdot \vec{r}$. Hence only the nonlinear coupling term $-(b+d)(\nabla \phi)^{2}$ reflects the diffusion effect on frequency in Eq. (11). The interaction between phase and amplitude modes yields the nonlinear coupling term from linear diffusive coupling. In this sense, simple phase models such as coupled circle map [18] cannot account for the results presented in this letter since it does not have amplitude variables.

[20] The term $-(b+d)(\nabla \phi)^{2}$ is always positive since $b+d<0$ from the experimental consideration explained near Eq. (3). Note that labyrinths would arise at the lower frequency if we chose the sign of $b, d$ positive. In such a case, diffusion decreases the frequency of patterns, enhancing locking at low frequencies. 


\section{FIGURES}

FIG. 1. Phase diagram : frequency deviation $\nu$ versus critical forcing amplitude $c$. (a) $n=1$, (b) $n=2$, (c) $n=3$. Solid lines are critical amplitude $c_{l o}$ for a single oscillator. The shaded regions are obtained from the numerical integration of Eq. (11) $1 \mathrm{~F}$ : flat, $2 \mathrm{~F}: \pi$ fronts, $3 \mathrm{~F}: 2 \pi / 3$ fronts, BD: chaotically bursting domains, LABY: labyrinthine patterns, TA:targets. See the text for details.

FIG. 2. Numerical integration results, Plot of $\operatorname{Re}(\psi)$, (a) flat $(n=1, \nu=-0.4, c=0.09)$. (b) $\pi$ fronts $(n=2, \nu=-0.1, c=0.4)$. (c) $2 \pi / 3$ fronts $(n=3, \nu=-0.1, c=0.45)$. (d) labyrinths $(n=2, \nu=0.1, c=0.4)$. (e) target $(n=3, \nu=-0.15, c=0.32)$. (f) chaotically bursting domains $(n=3, \nu=-0.65, c=0.26)$.

FIG. 3. (a) Time series at one point in Fig 2(c). (b) Parametric plot of real and imaginary parts of $\psi$ in a complex plane corresponding to Fig.2(c). The abscissa is $\operatorname{Re}(\psi)$ and the ordinate is $\operatorname{Im}(\psi)$.

FIG. 4. (a) Chaotic time series at one point in Fig.2(f). (b) Time evolution of $(\nabla \phi)^{2}$ at the same point. 

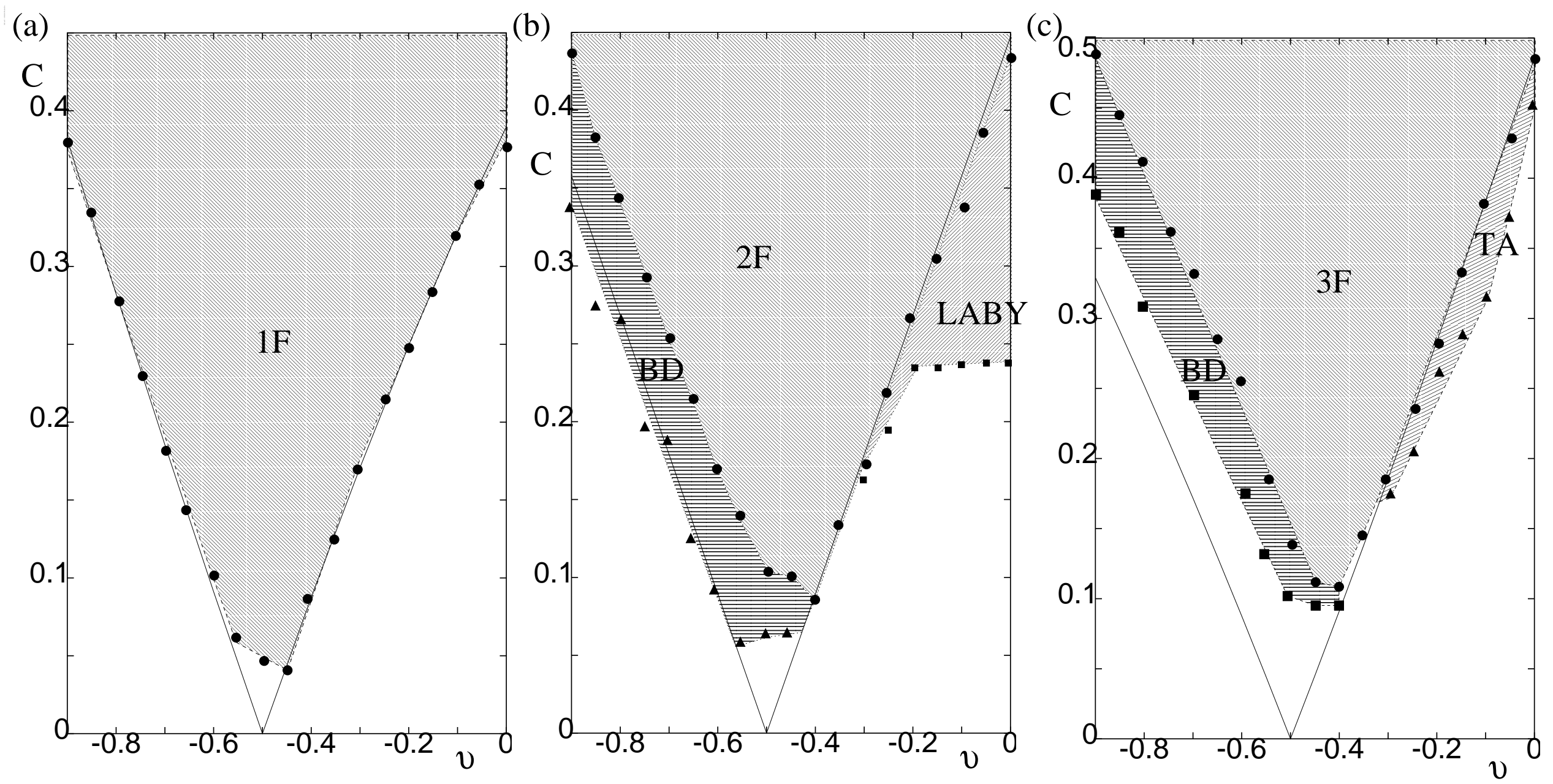
This figure "0101003.fig2.jpg" is available in "jpg" format from: http://arxiv.org/ps/nlin/0101003v2 


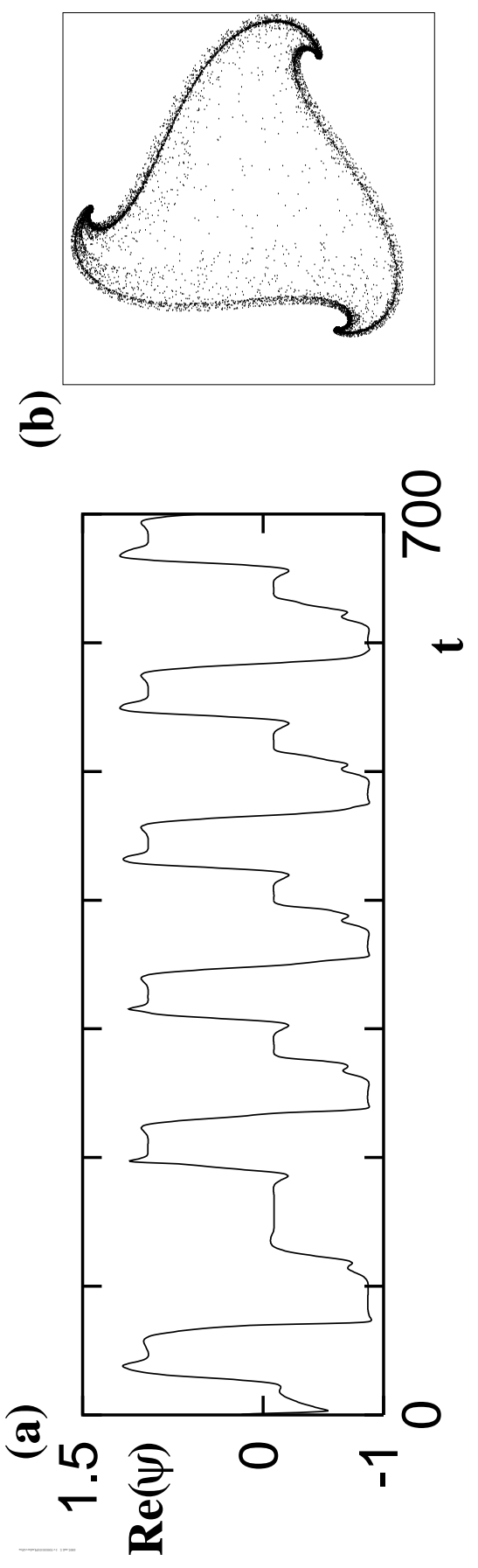


(a) 1.5

$\operatorname{Re}(\psi)$

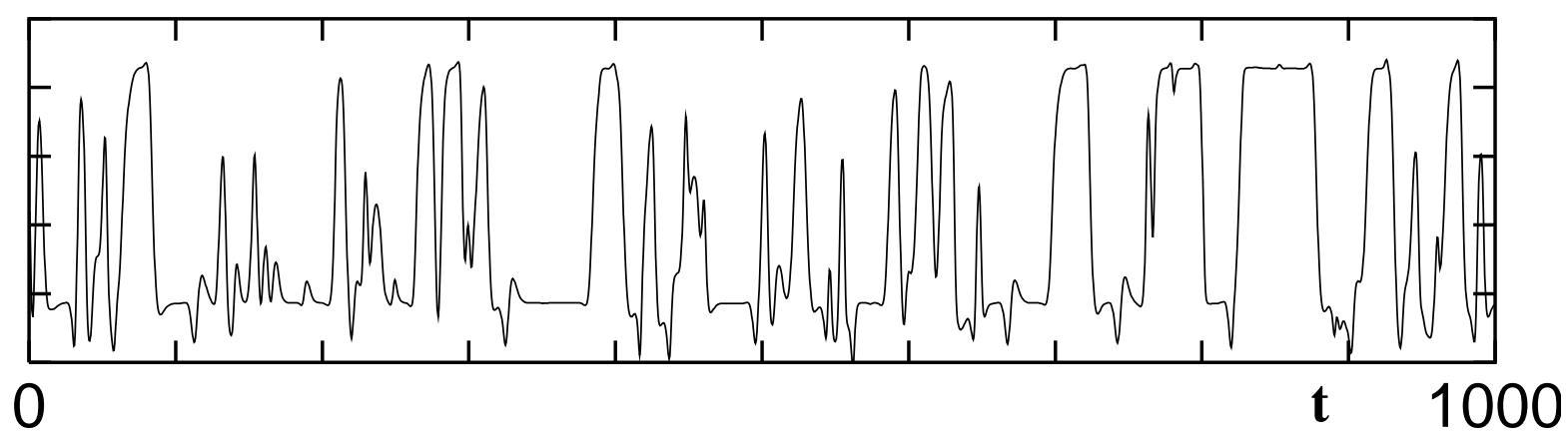

(b) 1.5

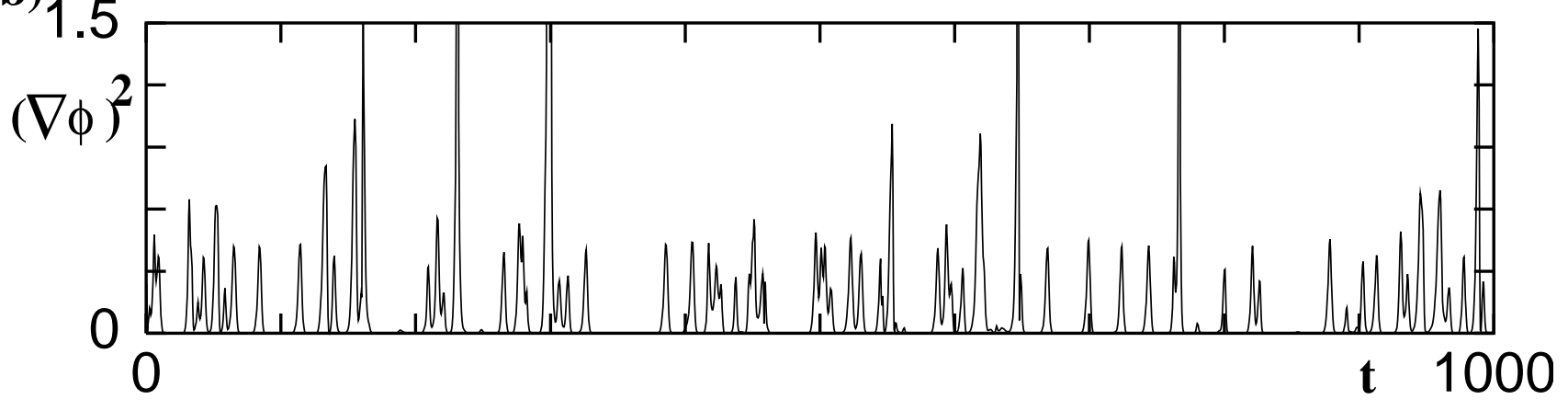

